\title{
AVALIAÇÃO DO POTENCIAL DA CRIAÇÃO DE CORVINA (Micropogonias furnieri) EM TANQUE- REDE NO ESTUÁRIO DA LAGOA DOS PATOS, BRASIL
}

\author{
LUÍS ANDRÉ SAMPAIO, DENÍLSON BURKERT, FELIPE MORAIS SANTOS, DANILO PEDRO STREIT JÚNIOR, MARCELO BORGES \\ TESSER \\ Universidade Federal do Rio Grande, Instituto de Oceanografia, Laboratório de Piscicultura Estuarina e Marinha, CP 474, Rio Grande-RS
} CEP 96201-900. Brasil. sampaio@mikrus.com.br

\section{RESUMO}

O estuário da Lagoa dos Patos é considerado um local adequado para a aquacultura por ser um ecossistema protegido, favorecendo a criação em tanques-rede e cercados. A viabilidade da produção da corvina (Micropogonias furnierı) em tanquerede ainda não foi determinada, portanto este trabalho foi realizado com o objetivo de estudar o seu crescimento em condições de clima quente e frio. Foram utilizados tanques-rede de $3 \times 3 \times 1,2 \mathrm{~m}$ (comprimento $\times$ largura $\times$ altura) instalados junto a um trapiche na enseada do Saco do Justino no estuário da Lagoa dos Patos (Rio Grande - RS). A sobrevivência dos juvenis de corvina foi $76 \%$ para o período de frio, enquanto no quente foi de $67 \%$. Ao final do período de criação de frio obteve-se taxa média de crescimento específico de $0,40 \% /$ dia, enquanto no quente o crescimento foi de $0,81 \% / d i a$. A criação de juvenis de corvina em tanque-rede no estuário pode ser praticada o ano todo, mas é importante buscar informações para aprimorar sua taxa de sobrevivência e o seu crescimento, no período de frio.

PALAVRAS CHAVE: gaiola, piscicultura, salinidade, Sciaenidae, temperatura

\section{ABSTRACT}

Evaluation of cage culture of whitemouth croaker (Micropogonias furnieri) in the Patos Lagoon estuary, Brazi The Patos Lagoon estuary is considered a suitable aquaculture site because it is a sheltered ecosystem, which favors cage and pen rearing. The viability of whitemouth croaker (Micropogonias furnieri) production in cages has not been investigated. Therefore, this work had the objective to evaluate growth and survival of M. furnieri in rearing conditions in the wild, in cold and warm seasons. Cages ( $3 \times 3 \times 1.2 \mathrm{~m}$; length $\mathrm{x}$ width $\mathrm{x}$ height) were installed near a pier at the Saco do Justino inlet in the Patos Lagoon estuary (Rio Grande- RS). Whitemouth croaker survival during the cold season was $76 \%$, while during warm season it was $67 \%$. At the end of the winter rearing period a specific growth rate of $0.40 \% /$ day was achieved, while during the warm it averaged $0.81 \% /$ day. Estuarine cage culture of whitemouth croaker can be done all year round, but it is important to improve survival and growth, during the cold season.

KEYWORDS: cage, fish culture, salinity, Sciaenidae, temperature

\section{INTRODUÇÃO}

A criação de peixes em tanque-rede é uma atividade zootécnica sendo desenvolvida em ritmo acelerado em diversas partes do mundo. Além das técnicas tradicionais de criação de peixes de água doce (Huchette \& Beveridge 2003), a produção de peixes marinhos em tanque-rede também vem recebendo atenção nos últimos anos (Petridis \& Rogdakis 1996).

No Brasil, a piscicultura em tanque-rede está bastante desenvolvida em ambientes de água doce (Chellappa et al. 1995). Por outro lado, a produção comercial de peixes marinhos e/ou estuarinos, independente do sistema adotado, é nula (Ibama 2005). Os registros de cultivo de peixes marinhos no Brasil são dominados por estudos sobre a reprodução e larvicultura (Cerqueira \& Tsuzuki, 2008; Sampaio et al. 2008), sendo poucos os registros sobre engorda (Sanches et al. 2007; Sampaio et. al. 2011).

A Lagoa dos Patos é considerada um local adequado para a aquacultura por ser um ecossistema protegido, favorecendo a criação em tanques-rede e cercados (Soares et al. 2005). No entanto, esse ambiente está sujeito a grandes variações ambientais
(Baumgarten \& Niencheski 1990) sendo necessário avaliar o potencial de produção de diferentes espécies em condições extremas de temperatura e salinidade, fatores físico-químicos de grande variação sazonal.

A corvina Micropogonias furnieri é uma espécie demersal com distribuição desde as Antilhas até a Argentina que apresenta grande importância para a pesca comercial e artesanal (Haimovici et al. 1997). No entanto, Vasconcelos e Haimocivi (2006) citam que a espécie encontra-se sobre explotada.

Estudos sobre a sua reprodução (Berois et al. 2004; Albuquerque et al. 2009) apontam para a viabilidade de produção de fases jovens em laboratório. Já Aristizabal-Abud (1992) cita que juvenis de corvina são eurialinos e esses peixes apresentam menor consumo de oxigênio em salinidades entre 17 e 19.

Assim, esse estudo foi realizado para avaliar a potencialidade da utilização da Lagoa dos Patos para a criação da corvina (Micropogonias furnierı), espécie naturalmente encontrada na região costeira do Atlântico Sul Ocidental, durante o período quente e frio. 


\section{MATERIAIS E MÉTODOS}

\section{Estrutura física dos tanques-rede}

Tanques-rede $(3 \times 3 \times 1,2 \mathrm{~m}$; comprimento $\times$ largura $x$ altura) foram instalados junto a um trapiche na enseada do Saco do Justino no estuário da Lagoa dos Patos (Rio Grande - RS). Esses tanques-rede foram confeccionados no Laboratório de Piscicultura Estuarina e Marinha da FURG, sendo compostos por uma panagem de rede de poliéster revestido de PVC com malha de $8 \mathrm{~mm}$. Os tanques-rede foram cobertos por uma rede de poliamida com malha de $12 \mathrm{~mm}$, com objetivo de impedir a fuga dos peixes, bem como evitar a entrada de possíveis predadores.

Os tanques-rede foram fixados em varas de eucalipto ( $3 \mathrm{~m}$ de comprimento) enterradas a aproximadamente um metro de profundidade no sedimento, com uma distância de $3 \mathrm{~m}$ entre elas. Também foram colocadas varas de bambu de $4 \mathrm{~m}$ de comprimento amarradas com arame galvanizado perpendicularmente às varas de eucalipto, de modo a formar seis quadrados, sendo que em seu interior estavam dispostos os tanques-rede. Os tanques-rede foram amarrados a estes quadrados de bambus com fio de poliamida 210/24. Garrafas plásticas descartáveis de refrigerante (PET) de dois litros cheias de areia foram amarradas em cada um dos vértices inferiores dos tanques-rede para manter suas laterais distendidas permanentemente, evitando que houvesse redução de seu volume.

\section{Coleta dos peixes}

Os juvenis de corvina foram capturados no estuário da Lagoa dos Patos, logo após a coleta os peixes foram levados ao laboratório, onde foram mantidos em tanques de fibra de vidro de $1000 \mathrm{~L}$ por aproximadamente trinta dias até a estocagem nos tanques-rede. Durante esse período os peixes receberam 3 banhos profiláticos de formol (100 ppm) durante uma hora. Os peixes foram alimentados com ração extrusada (Purina, Camaronina 35HP; proteína $35 \%$, extrato etéreo $8 \%$, cinza $12 \%$ e umidade $13 \%$ segundo o fabricante) à vontade. Diariamente o tanque era sifonado para retirada de restos alimentares e fezes e a água totalmente renovada.

\section{Estocagem e manejo diário}

Dois períodos distintos de estocagem foram realizados, sendo denominados de período de criação de frio e de quente. Para o período de frio, os tanques-rede foram povoados no final de abril e mantidos até o final de setembro de 1999. Quatro tanques-rede foram estocados com corvinas ( $n=30$, 30,31 e 35) com peso inicial médio de $12,1 \mathrm{~g} \pm 0,3$ (média \pm erro padrão). A criação do período de quente teve início no final de novembro de 1999, se estendendo até o início de junho de 2000. Dois tanques-rede foram estocados com corvinas $(n=18 \mathrm{e}$ 21), peso médio de $57,8 \mathrm{~g} \pm 2,88$ (média \pm erro padrão).

Os dados de temperatura e salinidade foram registrados diariamente pela manhã por meio de termômetro de mercúrio e refratômetro de mão. O alimento foi oferecido diariamente pela manhã, na forma de ração extrusada (Purina, Camaronina 35HP; proteína $35 \%$, extrato etéreo $8 \%$, cinza $12 \%$ e umidade $13 \%$ segundo o fabricante) com taxa de arraçoamento entre 8 a $10 \%$ do peso vivo ao dia. No período de quente, os peixes foram alimentados diariamente com a mesma ração, mas o alimento foi oferecido em excesso. Em ambos os períodos houve sobra de alimentos que infelizmente não foram mensuradas.

Para avaliar o crescimento dos peixes foram feitas biometrias (14 durante o período de quente e 7 durante o período de frio), nas quais todo o plantel de peixes de cada tanque-rede foi amostrado. Os peixes foram anestesiados com benzocaína 50ppm e em seguida o peso foi registrado com balança eletrônica (precisão de $0,1 \mathrm{~g}$ ) e o comprimento com um ictiômetro (precisão de 0,1 cm). Os peixes foram devolvidos para os tanques-rede de origem após as biometrias.

Foram calculados: a taxa de sobrevivência: $\mathrm{S}=$ (nf-ni/ni) x 100 (onde nf é o número final de peixes e ni é o número inicial de peixes); a taxa de crescimento específica diária: $\mathrm{G}=[(\ln \mathrm{pf}-\ln \mathrm{pi}$ ) $/ \Delta \mathrm{t}] \times 100$ (onde pf é 0 peso médio final, pi é o peso médio inicial e $\Delta$ t é o tempo em dias); e o fator de condição de Fulton: $F C=P / C^{3}$ (onde $\mathrm{P}$ é o peso e $\mathrm{C}$ é o comprimento).

\section{RESULTADOS}

No período de frio a temperatura média foi de $16 \pm 2^{\circ} \mathrm{C}$ com valores oscilando entre 8 e $21^{\circ} \mathrm{C}$ enquanto que a salinidade média observada foi de $7 \pm$ 
$6 \%$ com valores entre 0 (zero) e $18 \%$ (Figura $1 \mathrm{~A}$ ). No quente os valores de temperatura e salinidade registrados mostraram declínio ao longo do tempo, entretanto houve um pico de salinidade durante o mês de maio. A temperatura média foi de $23 \pm 2{ }^{\circ} \mathrm{C}$ com valores entre 14 e $33^{\circ} \mathrm{C}$ e a salinidade foi de $22 \pm$ $5 \%$ com valores entre $8 \%$ e $31 \%$ (Figura 1B).
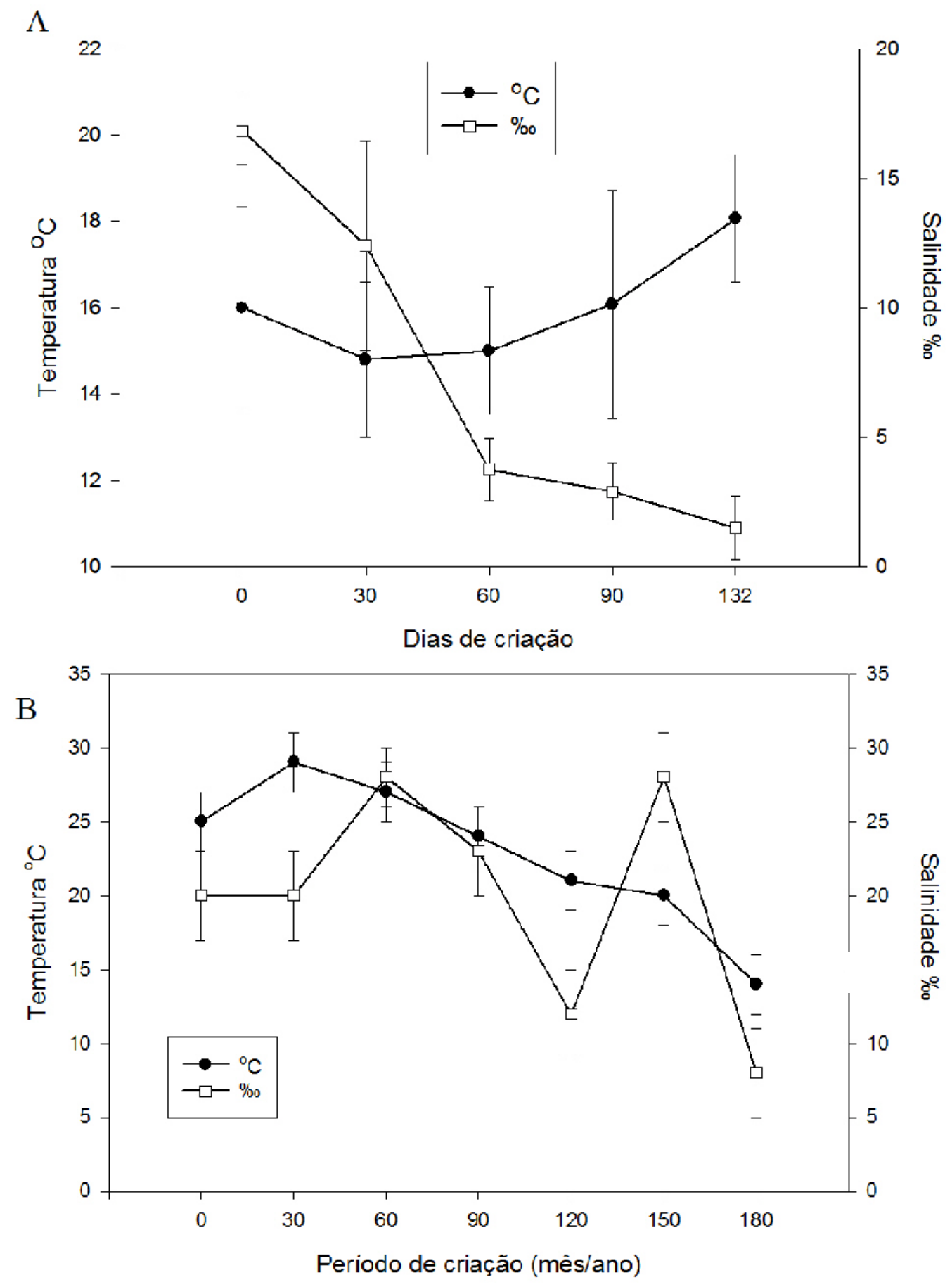

FIGURA 1 - Valores médios ( \pm erro padrão) da temperatura $(-\bullet)$ e da salinidade $(\longrightarrow-)$ nos tanques-rede durante o período de frio (A) e de quente $(B)$. 


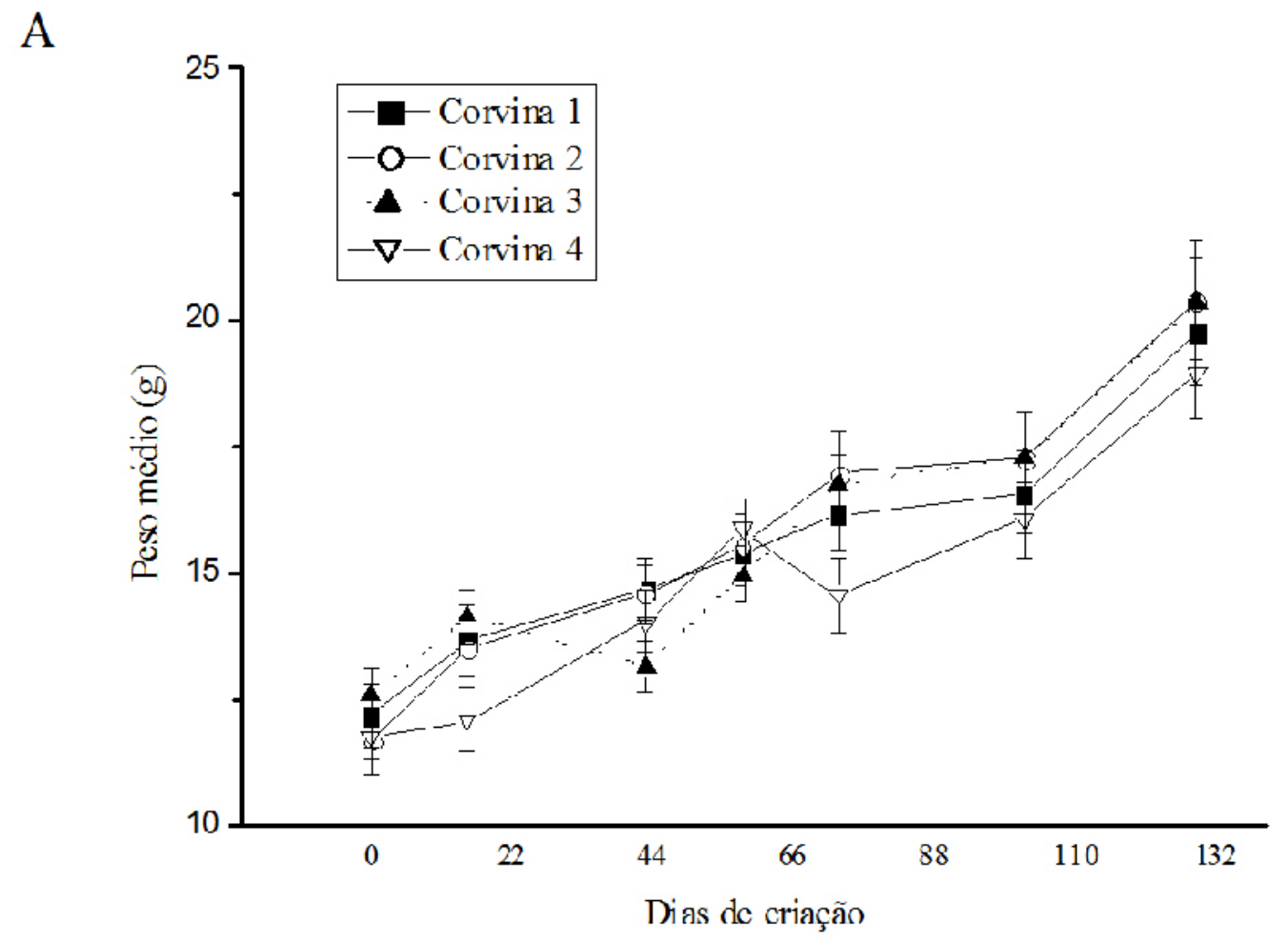

B

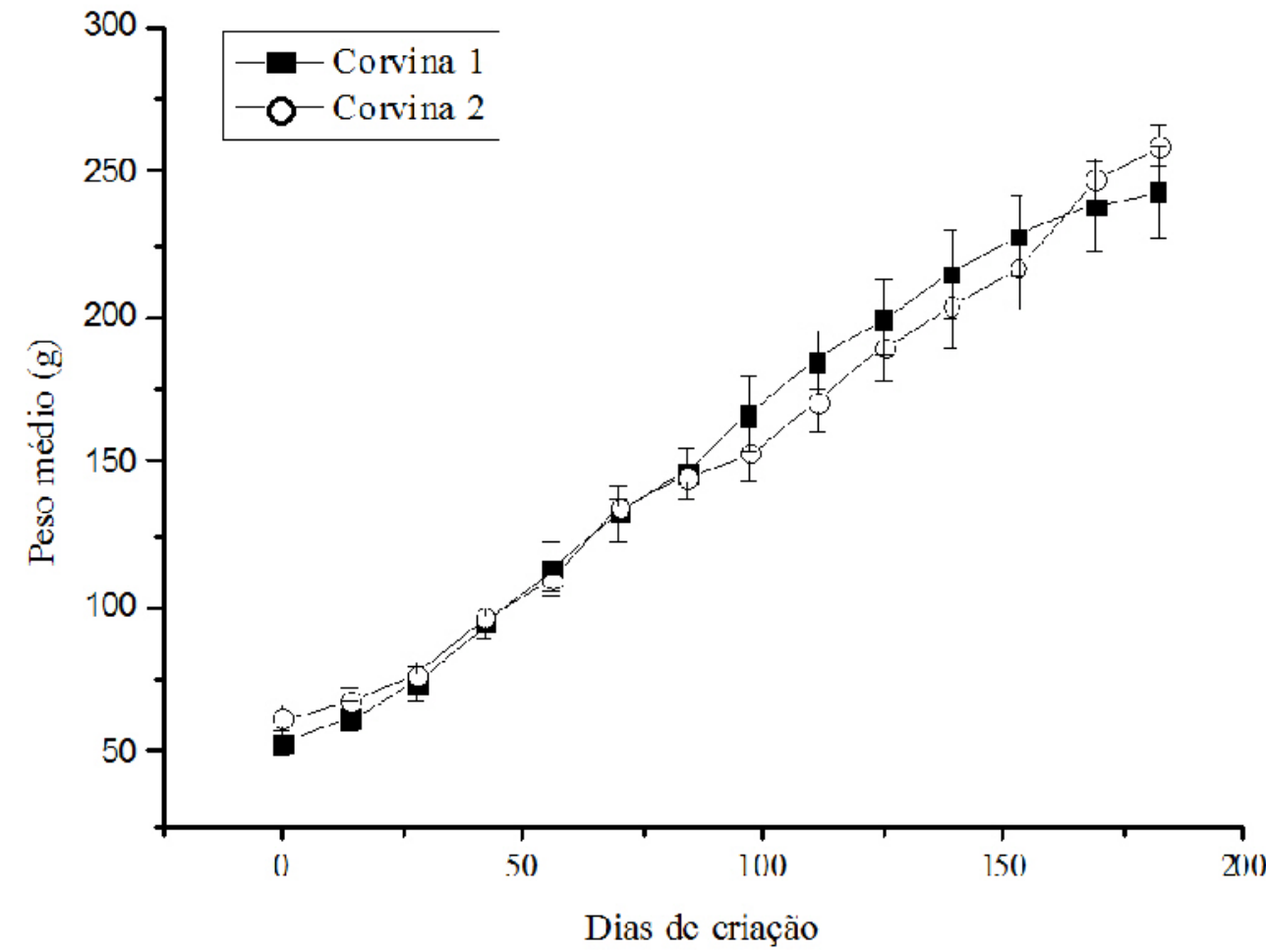

FIGURA 2 - Peso médio ( \pm erro padrão) dos juvenis de corvina Micropogonias furnieri criados no estuário da Lagoa dos Patos durante o período de frio $(A)$ e de quente $(B)$. Cada linha representa os peixes de um tanque-rede. 
A sobrevivência dos juvenis de corvina no frio foi de $76 \%$, enquanto no quente foi de $67 \%$. Ao final do período de frio obteve-se o peso médio final de 19,9 \pm $0,3 \mathrm{~g}$ (Figura $2 \mathrm{~A}$ e Tabela 1). No quente, o crescimento em peso dos juvenis de corvina de ambos os tanquesrede foi constante, mostrando um comportamento similar, atingindo o peso médio final de $251,8 \pm 43,0 \mathrm{~g}$ (Figura 2B e Tabela 1). Os valores de taxa de crescimento específico e do fator de condição durante o período de quente foram superiores aos valores encontrados para o período de frio (Tabela 1).

TABELA 1 - Valores médios (média \pm erro padrão da média) para sobrevivência (S), taxa de crescimento específico diário $(G)$, fator de condição ( $F C)$, comprimento (comp.) e peso médio inicial e final para juvenis de corvina Micropogonias furnieri criados em tanque-rede nos períodos de frio e quente.

\begin{tabular}{cccccccc}
\hline Período & $\begin{array}{c}\mathrm{S} \\
(\%)\end{array}$ & $\begin{array}{c}\mathrm{G} \\
(\% / \mathrm{dia})\end{array}$ & $\mathrm{FC}$ & $\begin{array}{c}\text { Comp. Inicial } \\
(\mathrm{cm})\end{array}$ & $\begin{array}{c}\text { Comp. Final } \\
(\mathrm{cm})\end{array}$ & $\begin{array}{c}\text { Peso inicial } \\
(\mathrm{g})\end{array}$ & $\begin{array}{c}\text { Peso final } \\
(\mathrm{g})\end{array}$ \\
\hline Frio & $76 \pm 1$ & $0,40 \pm 0,02$ & $1,12 \pm 0,02$ & $10,0 \pm 0,1$ & $12,1 \pm 0,1$ & $12,08 \pm 0,3$ & $19,03 \pm 0,3$ \\
\hline Quente & $67 \pm 1$ & $0,81 \pm 0,03$ & $1,39 \pm 0,03$ & $17,1 \pm 0,3$ & $26,7 \pm 0,3$ & $57,82 \pm 2,88$ & $251,8 \pm 43$
\end{tabular}

\section{DISCUSSÃo}

O estuário da Lagoa dos Patos apresenta uma íntima relação com o sistema marinho e a planície costeira adjacente. Esse é um ecossistema aquático de alta produtividade natural, devido ao aporte de nutrientes e matéria orgânica provenientes dos corpos d'água continentais. As espécies de peixes que costumam frequentar este tipo de ambiente se beneficiam das condições abrigadas e da disponibilidade de alimentos no estuário e suas enseadas, favorecendo a sua sobrevivência e o seu crescimento (Castello \& Abreu 1998).

No frio, constatou-se que as corvinas estiveram sujeitos a baixas salinidades e temperaturas por um longo período. Esta combinação pode ter inibido o crescimento, o que já foi observado por Castello (1986) para corvinas em seu habitat natural (Estuário da Lagoa dos Patos). Peterson et al. (1999) afirmam que flutuações de salinidade podem resultar em variações no crescimento de Micropogonias undulatus em curto período de tempo. Milstein et al. (2000) trabalhando com o crescimento de pacu (Piaractus mesopotamicus) em tanques escavados sob baixas temperaturas verificaram que estes peixes perderam peso, no entanto a sobrevivência foi alta (98\%), sugerindo que esses peixes quando expostos a esta condição reduzem o seu metabolismo e consequentemente reduzem o consumo de alimento.

A corvina ( $M$. furnieri) parece adaptar-se bem às variações sazonais de temperatura e salinidade observadas no estuário da Lagoa dos Patos durante o ano. Por se tratar de uma espécie estuarino dependente (Vieira et al. 1998), que utiliza esse ecossistema para o seu desenvolvimento, ela pode apresentar boa adaptação fisiológica às variações ambientais. Os juvenis de corvina apresentaram a menor sobrevivência durante o período de quente, com $67 \%$ dos indivíduos vivos ao final do experimento, valor próximo ao encontrado durante o período de frio que foi de $76 \%$. No entanto, o presente estudo não pode determinar se houve relação entre a salinidade e a temperatura encontrada nos diferentes períodos experimentais sobre a sobrevivência. Desta forma, novas investigações devem ser realizadas para avaliar a causa da mortalidade das corvinas criadas em tanque-rede.

De acordo com Mcginty \& Rakocy (1999), a ração utilizada em tanques-rede deve ser totalmente balanceada para atender as exigências nutricionais da espécie. A dieta utilizada nesse estudo é formulada para a nutrição de camarões marinhos, pois ainda não existem rações comercias para peixes marinhos no Brasil. Outro fator a ser considerado é o crescimento dessa espécie no ambiente natural. Castello (1986) avaliou o crescimento da corvina neste estuário e verificou que o crescimento é reduzido, pois indivíduos com idade entre 1 e 2 anos apresentam comprimento de $17 \mathrm{~cm}$, atingindo $24 \mathrm{~cm}$ apenas ao completarem 4 anos. Este autor propôs duas hipóteses para o crescimento lento: a primeira cogita a emigração dos indivíduos maiores para o 
oceano adjacente; e a segunda considera os elevados custos energéticos para manutenção da homeostase em um ambiente com grandes variações de salinidade. As corvinas no presente trabalho cresceram de 17 a $26 \mathrm{~cm}$ em 6 meses, portanto a primeira hipótese parece ser a mais acertada, já que confinados em um tanque-rede os peixes não puderam deixar o estuário, e o crescimento foi maior do que na natureza.

Uma das espécies de peixes marinhos mais criada em tanque-rede na Europa é Sparus aurata, são necessários aproximadamente 190 dias para estes peixes crescerem entre 50 a $250 \mathrm{~g}$ (Petridis \& Rogdakis 1996), o que é equivalente a uma taxa de crescimento de $0,84 \% / d i a$, valor similar ao observado para $M$. furnieri no quente. $\mathrm{O}$ crescimento de Piaractus brachypomus, uma espécie de água doce, criada em gaiolas flutuantes com diferentes taxas de arraçoamento foi de 0,70\%/dia (Granado, 1996), sendo que esta é uma espécie considerada promissora para a piscicultura continental na América do Sul (Saint-Paul 1986). Isso demonstra o bom crescimento de juvenis de corvina durante o período quente.

O fator de condição de $M$. furnieri criada em tanque-rede é superior ao de exemplares selvagens que habitam o estuário da Lagoa dos Patos, pois Castello (1986) determinou valores de 1,1 no início do outono e 1,0 no início da primavera. Sendo o fator de condição um índice de saúde dos peixes, pode-se considerar que a produção de corvinas em tanque-rede aparentemente não prejudica seu estado nutricional. Entretanto, seria interessante investigar se a maior proporção de peso dos peixes produzidos em cativeiro se deve ao acúmulo de gordura ou de massa muscular.

A pesca do camarão Farfantepeneaus paulensis é bastante intensa no estuário da Lagoa dos Patos. Esta pescaria tem um forte impacto sobre a população de corvinas, pois segundo Vieira et al. (1996) aproximadamente 4,2 milhões de juvenis desta espécie são pescados por ano. O aproveitamento destes juvenis que seriam mortos durante a pesca do camarão para criação em tanque-rede poderia reduzir em parte o impacto sobre essa população ou até mesmo oferecendo um destino mais adequado para esse recurso.

\section{AGRADECIMENTOS}

L. A. Sampaio agradece ao CNPq pela Bolsa de Produtividade em Pesquisa (Processo n. 308013/2009-3). D. Burkert e F.M. Santos agradecem à FAPERGS e M.B. Tesser e D.P. Streit Junior agradecem ao CNPq pelas Bolsas de Iniciação Científica recebidas durante a realização deste trabalho.

\section{REFERÊNCIAS}

ALBUQUERQUE, C.Q., J. H., MUELBERT, L. A., SAMPAIO. 2009. Early developmental aspects and validation of daily growth increments in otoliths of Micropogonias furnieri (Pisces, Sciaenidae) larvae reared in laboratory. Pan-American Journal of Aquatic Sciences, 4(3): 259-266.

ARISTIZABAL-ABUD, E. O. 1992. Effects of salinity and weight on routine metabolism in the juvenile croaker, Micropogonias furnieri (Desmarest 1893), Journal of fish Bilogy, 40: 471-472.

BAUMGARTEN, M.G.Z. \& L.F. NIENCHESKI. 1990. O estuário da Laguna dos Patos: variações de alguns parâmetros físicoquímicos da água e metais associados ao material em suspensão. Ciência e Cultura, 42: 390-396.

BEROIS, N., BOLATTOM C., BRAUER, M.M., BARROS, C. 2004. Gametogenesis, histological gonadal cycle and in vitro fertilization in the whitemouth croaker (Micropogonias furnieri, Desmarest, 1823). Journal of Applied Ichthyology, 20: 169-175.

CASTELLO, J. P. \& P.C. ABREU. 1998. Interações entre os Ambientes Estuarino e Marinho. In: SEELIGER, U.; C. ODEBRECHT, J.P. CASTELLO. Os Ecossistemas Costeiros e Marinhos do Extremo Sul do Brasil. Ecoscientia, Brasil. Cap. 8: $199-203$.

CASTELLO, J.P. 1986. Distribución, crecimiento y maduración sexual de la corvina juvenil (Micropogonias furnierı) en el estuario de la "Lagoa dos Patos", Brasil. Physis, 44: 21-36.

CERQUEIRA, V.R. \& M.Y. TSUZUKI. 2008. A Review of spawning induction, larviculture and juvenile rearing of the fat snook, Centropomus parallelus. Fish Physiology and Biochemistry, DOI 10.1007/s10695-008-9245-y.

CHELLAPA, S., N.T. CHELLAPA, W.B. BARBOSA, F.A. HUNTINGFORDF \& M.C.M. BEVERIDGE. 1995. Growth and production of the Amazonian tambaqui in fixed cages under different feeding regimes. Aquaculture Internacional, 3: 11 - 21.

GRANADO, A. 1996. Efecto de la ración alimenticia sobre el crecimiento del morocoto, Piaractus brachypomus, (Osteichthyes: Characiformes) cultivado en jaulas flotantes. Asociación Latinoamericana de Acuicultura, 307 - 310.

HAIMOVICI M., J.P., CASTELLO, C.M., VOOREN. 1997. Fisheries. In: SEELIGER U., ODEBRECHT C., CASTELLO J.P. (Eds.), The Coast and Sea in the South-Western Atlantic. A Profile of Subtropical Convergence Environments. Verlag, New York: 183-196.

HUCHETTE S.M.H. \& M.C.M BEVERIDGE. 2003. Technical and economical evaluation of periphyton-based cage culture of tilapia (Oreochromis niloticus) in tropical freshwater cages. Aquaculture, 218: 219-234.

IBAMA. 2005. Estatística da Pesca 2004. Ministério do Meio Ambiente, 98.

MCGINTY, A.S. \& J.E. RAKOCY. 1999. Cage culture of tilapia. Southern Regional Aquaculture Center. Publication No. 281. 
MILSTEIN, A. 2000. Low temperature tolerance of pacu, Piaractus mesopotamicus. Environmental Biology of Fishes, 58: 455-460.

PETERSON, M.S., B.H., COMYNS, C. F., RAKOCINSKI, G.L., FULLING, 1999. Does salinity affect somatic growth in early juvenile Atlantic croaker, Micropogonias undulatus (L.)? Journal of Experimental Marine Biology and Ecology, 238: 199-207.

PETRIDIS, D. \& I. ROGDAKIS. 1996. The development of growth and feeding equations for sea bream, Sparus aurata L., culture. Aquaculture Research, 27: 413-419.

SAMPAIO, L. A., C. B. MOREIRA, K. C. MIRANDA-FILHO, A. N. ROMBENSO. 2011. Culture of cobia Rachycentron canadum (L) in near-shore cages off the Brazilian coast. Aquaculture Research, 42: 832-834.

SAMPAIO, L.A., R.B., ROBALDO, A, BIANCHINI,. 2008. Hormone induced ovulation, natural spawning and larviculture of Brazilian flounder Paralichthys orbignyanus (Valenciennes, 1839). Aquaculture Research, 39:712-717.

SANCHES, E.G., V.G. AZEVEDO \& M.R. COSTA. 2007. Criação da garoupa-verdadeira Epinephelus marginatus (Lowe, 1834) (Teleostei, Serranidae) alimentada com rejeito de pesca e ração úmida em tanques-rede. Atlântica, 29:121-126.
SAINT-PAUL, U. 1986. Potential for aquaculture of South American freshwater fishes: A review. Aquaculture, 54: $205-240$.

SOARES, R.T., S. PEIXOTO, W. WASIELESKY \& F. D'INCAO. 2005. Feeding rhythms and diet of Farfantepenaeus paulensis under pen culture in Patos Lagoon estuary, Brazil. Journal of Experimental Marine Biology and Ecology, 322: 167-176.

VASCONCELOS, M., M, HAIMOVICI. 2006. Status of white croaker Micropogonias furnieri exploited in southern Brazil according to alternative hypotheses of stock discreetness. Fisheries Research, 80: 196-202.

VIEIRA, J.P. J.P. CASTELLO \& L.E. PEREIRA. 1998. Ictiofauna. In: SEELIGER, U., C. ODEBRECHT \& J.P. CASTELLO (ed.) Os Ecossistemas Costeiros e Marinhos do Extremo Sul do Brasil. Ecoscientia, Brasil, Cap. 4.13: 60-66.

VIEIRA, J.P., M.C. VASCONCELLOS, R.E.E. SILVA \& L.G.F. FISCHER. 1996. A rejeição da pesca do camarão-rosa (Penaeus paulensis) no estuário da Lagoa dos Patos, RS, Brasil. Revista Atlântica, 18: 123-142.

Submetido - 16/03/2009

Aceito - 06/10/2010 
